\title{
Why the P600 is not just a P300: the role of the basal ganglia
}

\author{
Stefan Frisch $^{\mathrm{a}, \mathrm{b}, *}$, Sonja A. Kotz ${ }^{\mathrm{b}}$, D. Yves von Cramon ${ }^{\mathrm{b}}$, Angela D. Friederici ${ }^{\mathrm{b}}$ \\ ${ }^{\mathrm{a}}$ Institute of Linguistics, University of Potsdam, PO Box 6015 53, D-14415 Potsdam, Germany \\ ${ }^{\mathrm{b}}$ Max-Planck-Institute of Cognitive Neuroscience, Leipzig, Germany
}

Accepted 23 October 2002

\begin{abstract}
One of the important issues in event-related brain potential research is whether the language-related P600 and the P300 oddball effect are distinct components or not. We addressed this question by testing 14 aphasic patients, half of them with lesions including the basal ganglia and half of them with temporo-parietal lesions, in both an auditory oddball task and an experiment with auditory presented verb inflection violations. Whereas both patient groups displayed a clear P300 effect in the oddball experiment, only the group with temporo-parietal lesions showed a P600 in the language experiment. These data indicate that the basal ganglia seem to play a crucial role in the modulation of the P600, but not of the P300 component.
\end{abstract}

(C) 2002 Elsevier Science Ireland Ltd. All rights reserved.

Keywords: Event-related potentials (ERPs); P300; P600; Basal ganglia; Language processing; Oddball

\section{Introduction}

Starting with the first reports, a centro-parietal positive event-related potential (ERP) component (P600) has been associated with processing efforts related to syntactic aspects of language (Osterhout and Holcomb, 1992; Hagoort et al., 1993). Meanwhile, similar positive ERP deflections have been found for a broad range of syntactic anomalies, such as phrase structure violations (Friederici et al., 1999; Hahne and Friederici, 1999), morphosyntactic violations (Hagoort et al., 1993; Gunter et al., 1997; Coulson et al., 1998a), argument-structure and case violations (Friederici and Frisch, 2000; Frisch and Schlesewsky, 2001), or dispreferred continuations of temporarily ambiguous sentences (Osterhout and Holcomb, 1992; Mecklinger et al., 1995). As a consequence, the P600 ERP component is taken as a specific marker of syntactic aspects of language processing (as opposed to the lexical-semantic N400 effect) by many authors in the field (Osterhout and Holcomb, 1992; Friederici, 1995).

There is, however, an ongoing debate as to whether the P600 is indeed language specific or just a P300-like effect, indicating the detection of an unexpected, task-relevant target (Gunter et al., 1997; Coulson et al., 1998a). The classical experimental paradigm to elicit a P300 or, more precisely, the P3b subcomponent of the P300 complex

\footnotetext{
* Corresponding author. Tel.: +49-331-9772986; fax: +49-331-9772087.

E-mail address: frisch@rz.uni-potsdam.de (S. Frisch).
}

(Picton, 1992) is the so-called auditory oddball paradigm. Subjects listen to a series of tones which can be classified into two different categories, with one of the two being taskrelevant (i.e. deviant tones have to be counted) and evoking a P300 response. The fact that the P3b and the P600 have a very similar centro-parietal scalp distribution and that the amplitudes of both increase when the number of critical targets decreases (Donchin, 1981; Coulson et al., 1998a; Hahne and Friederici, 1999) has led some authors to believe that the two components are identical (Coulson et al., 1998a). Latency differences between the P600 and the P300 oddball effect are explained with the higher complexity of linguistic stimuli.

Opponents of this identity hypothesis argued that probability dependence holds for other components as well and that due to the inverse problem similar scalp distributions can in principle not reveal that both components rely upon the same neuronal generators (Osterhout and Hagoort, 1999). However, all opponents seem to agree that different P300 and P600 generators would support existence of two different components (Coulson et al., 1998b; Osterhout and Hagoort, 1999). This question, however, has not been settled yet.

As for the P3b, it is assumed that this component reflects activity from a number of different sources (Picton, 1992; Johnson, 1993; Mecklinger et al., 1998), which seem to be differentially involved as a function of the modality of the stimulus (Johnson, 1989; Rogers et al., 1991). For the auditory P3b, Rogers and colleagues described two main genera- 
Table 1

Descriptions of lesions determined by MRI scans for each individual patient in both groups ${ }^{\mathrm{a}}$

\begin{tabular}{|c|c|c|c|c|c|c|}
\hline Patient group test (AUD) & Lesion site/left hemisphere & Classification & Age (years) & Sex & Token Test & AAT scores \\
\hline \multicolumn{7}{|c|}{ Patients with lesions including the basal ganglia } \\
\hline 1 & Fronto-lateral, insula, caud, put & Broca & 55 & $\mathrm{~F}$ & 5 & $47 / 60$ \\
\hline 2 & Put & Residual & 60 & M & 0 & $52 / 60$ \\
\hline 3 & Glob pall & Non-aphasic & 57 & M & - & - \\
\hline 4 & Caud, put & Amnesic & 50 & M & 27 & $46 / 60$ \\
\hline 5 & Fronto-lateral, insula, caud, put & Residual & 62 & M & 6 & $59 / 60$ \\
\hline 6 & Fronto-lateral, insula, caud, put & Amnesic & 38 & $\mathrm{~F}$ & 21 & $43 / 60$ \\
\hline 7 & Caud, put & Amnesic & 45 & M & 1 & $51 / 60$ \\
\hline \multicolumn{7}{|c|}{ Patients with lesions excluding the basal ganglia } \\
\hline 8 & Temporo-parietal-lateral & Amnesic & 61 & M & 15 & $50 / 60$ \\
\hline 9 & Temporo-parietal-lateral & Residual & 39 & M & 0 & $59 / 60$ \\
\hline 10 & Fronto-lateral, insula & Residual & 43 & M & 3 & $52 / 60$ \\
\hline 11 & Multiple (bilateral), white matter & Non-aphasic & 51 & $\mathrm{~F}$ & 0 & - \\
\hline 12 & Parieto-lateral & Non-aphasic & 50 & $\mathrm{~F}$ & 0 & - \\
\hline 13 & $\begin{array}{l}\text { Fronto-lateral, insula, thalamus } \\
\text { (bilateral) }\end{array}$ & Non-aphasic & 41 & M & 0 & - \\
\hline 14 & Temporo-parietal-lateral & Non-aphasic & 61 & M & 0 & - \\
\hline
\end{tabular}

a The severity of the language comprehension disorder is indicated by the number of mistakes in the Token Test: no/very mild disorder (0-6); light (7-23); severe $(>40)$. In addition, the auditory comprehension scores of the Aachen Aphasia Test (AAT) are listed for each patient (only patients with a Token Test score greater than 0 were tested with the AAT). The degree of the comprehension disorder is evaluated based on a total of 60 points. Abbreviations: caud $=$ caudatum, put $=$ putamen, glob pall $=$ globus pallidus.

tors, one in the thalamic region and one in the posterior temporal lobe (Rogers et al., 1991). Studies using ERPs and functional magnetic resonance imaging (fMRI) suggest an involvement of the thalamus, the anterior portion of the gyrus cinguli as well as portions of the supramarginal gyrus (Menon et al., 1997). To our knowledge, however, the neural basis of the P600 component has not been specified so far.

In the present studies, we want to address the question of whether the neuronal sources of the two components are identical by testing two groups of aphasic patients (with versus without lesions involving the basal ganglia) in two experiments: one using grammatical violations and the second an auditory oddball.

\section{Experiment 1}

In the first experiment, subjects listened to grammatical and ungrammatical sentences. Ungrammatical sentences contained morphosyntactic violations. According to a number of previous studies, this violation elicits a P600 component in the ERP (Gunter et al., 1997; Coulson et al., 1998a).

\subsection{Methods}

\subsubsection{Participants}

Fourteen brain damaged patients participated in the study after giving informed consent. Their lesion sites were determined by (T1- and T2-weigthed) MRI datasets from a 3.0 T system (Bruker 30/100 Medspec) and evaluated by an experienced neuroanatomist. The individual patient information is listed in Table 1.

\subsubsection{Materials}

All sentences were German passive constructions. In the incorrect sentences, the verb form was an infinitive instead of a participle, such as in Im Haus wurde oft streichen (literal translation: In the house it was often to paint), thereby creating a morphosyntactic violation compared to a sentence such as Im Garten wurde oft gearbeitet (literal translation: In the garden it was often worked). The critical words (verbs) in both conditions did not differ with respect to length and lemma frequency $(\mathrm{F}<1$ according to the CELEX database, Baayen et al., 1993). In order to exclude possible confounds with a sentence final wrap up effect (Friederici and Frisch, 2000), the critical verb form was always followed by 'und' (and) and a second verb which always had the correct form. Forty sentences per condition, resulting in 80 critical sentences were created on the basis of 80 noun-(intransitive) verb sets. In addition, 80 filler sentences (half of them ungrammatical) with a similar sentence structure were created.

All sentences were spoken by a female native speaker of German at a normal speech rate, recorded onto digital audiotape and digitized at a sampling rate of $44.1 \mathrm{KHz}$. In order to ensure a precise time locking of the ERP in each individual sentence, the onset of the critical word was marked by way of a careful inspection of the auditory and visual signal.

\subsubsection{Procedure}

All 160 sentences were presented auditorily via loud speakers in a randomized order which was counterbalanced across subjects. Each sentence was introduced by a visual cue presented on the center of a computer screen. $800 \mathrm{~ms}$ 
patients with lesions

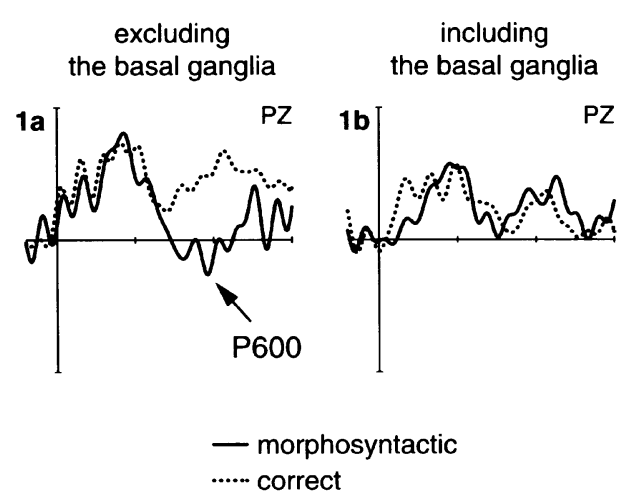

patients with lesions

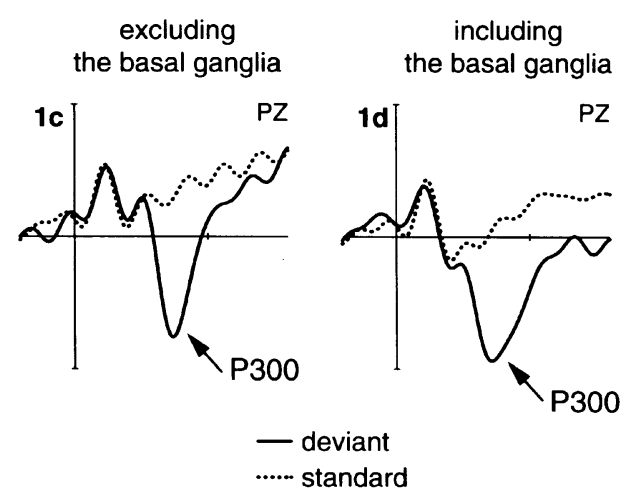

Fig. 1. ERP effects for both experiments for both patient groups at the electrode Pz. Negativity is plotted upwards and each tick on the $\mathrm{x}$-axis indicates 500 ms. ERPs from the onset of the critical verb in Experiment 1 (onset at $0 \mathrm{~ms} /$ vertical bar) up to $1500 \mathrm{~ms}$ show that the patients without basal ganglia lesions exhibit a clear P600 response (a); whereas no such effect is visible for the patients with lesions including the basal ganglia (b). ERPs relative to the onset of the target tones in Experiment 2 (onset at $0 \mathrm{~ms} / \mathrm{vertical}$ bar) up to $800 \mathrm{~ms}$ show a P300 for rare as compared to frequent targets for both the patients without (c); and for those with lesions including the basal ganglia (d).

after the offset of the sentence, subjects were asked to perform an acceptability judgment task. The next trial started $1000 \mathrm{~ms}$ after the subject's button press.

The electroencephalogram (EEG) was recorded from 19 scalp sites by means of $\mathrm{Ag} / \mathrm{AgCl}$ electrodes. $\mathrm{C} 2$ served as ground electrode and the left mastoid as on-line reference (recordings were re-referenced to linked mastoids off-line). Electrode impedances were kept below $5 \mathrm{kOhm}$. In order to control for eye movement artifacts, a horizontal and a vertical electrooculogram (EOG) were recorded. Individual EEG recordings were scanned for artifacts by means of a rejection algorithm as well as on the basis of visual inspection.

\subsubsection{Data analysis}

ERPs were computed for each subject and each electrode. All ERP averages were aligned to a $200 \mathrm{~ms}$ baseline before the onset of the adverb preceding the critical verb. Only trials with correct responses and without ocular and amplifier saturation artifacts entered the averages. The average percentage of trials rejected because of behavioral performance ${ }^{1}$ and artifacts was $21.4 \%$. The statistical analysis was computed in an analysis of variance (ANOVA) design with a within-subjects factor grammaticality (correct versus ungrammatical) and a between-subjects factor lesion (basal ganglia involved versus not involved) on the condition means in a time window between 700 and $1200 \mathrm{~ms}$. As clear effects were only visible over posterior sites, statistical analyzes were done for the midline electrode $\mathrm{Pz}$ and, sepa-

\footnotetext{
1 On average, subjects made $14.8 \%$ errors in the correct condition and $25.7 \%$ in the violation condition. This was reflected in a marginal main effect of grammaticality $(F(1,12)=3.55, P=0.08)$. Furthermore, there was a main effect of lesion $(F(1,12)=13.64, P<0.01)$ due to more errors for the group with lesions including the basal ganglia (28.0\%) as compared to the group without a basal ganglia lesion (12.5\%). There was no grammaticality $\times$ lesion interaction between both factors $(F(1,12)=3.10, P=0.10)$.
}

rately, for a left-posterior (electrodes $\mathrm{Cp} 5, \mathrm{P} 3, \mathrm{O} 1$ ) and a right-posterior (electrodes $\mathrm{Cp} 6, \mathrm{P} 4, \mathrm{O} 2)$ region of interest (ROI), including a factor hemisphere (left versus right).

\subsection{Results}

Figs. 1a,b display the ERP patterns from the onset of the critical verb up to $1500 \mathrm{~ms}$ thereafter for each of the two lesion groups at electrode Pz. Fig. 1a shows that the patients without basal ganglia lesions show a clear P600 for the morphosyntactic violation. As can be seen from Fig. 1b, the patients suffering from a lesion which involves the basal ganglia do not show such an effect.

The global ANOVA for the ungrammaticality effect in the P600 time window (700-1200 ms) at electrode Pz revealed a main effect of grammaticality $(F(1,12)=14.3$, $P<0.01)$ and an interaction of grammaticality $\times$ lesion $(F(1,12)=23.2, P<0.01)$ due to the fact that only the patients without a basal ganglia lesion showed a P600 grammaticality effect $(F(1,6)=68.1, P<0.01)$, but not the patients with such a lesion $(F<1)$.

Over the posterior ROIs, we also found a main effect of grammaticality $(F(1,12)=38.2, P<0.01)$ and an interaction of grammaticality $\times$ lesion $(F(1,12)=46.6$, $P<0.01)$. Again, only the patients with intact basal ganglia exhibited a P600 response $(F(1,6)=92.9, P<0.01)$, but not the patients with basal ganglia lesions $(F<1)$. No interactions with hemisphere were significant.

\section{Experiment 2}

The results of Experiment 1 suggest that the observation of a P600 component in response to a grammatical violations depends on intact basal ganglia. The goal of Experiment 2 was to test whether the basal ganglia also play a crucial role in generating or mediating of the $\mathrm{P} 3 \mathrm{~b}$ compo- 
nent elicited by improbable, but task-relevant tones (auditory oddball paradigm).

\subsection{Methods}

\subsubsection{Participants}

The same subjects as in Experiment 1 took part in Experiment 2 after having performed Experiment 1.

\subsubsection{Materials and procedure}

The auditory oddball task used a two-tone block. Subjects heard standard tones $(600 \mathrm{~Hz})$ with a probability of 0.8 and deviants $(660 \mathrm{~Hz})$, with a probability of 0.2 . The block contained a total of 500 auditory stimuli. All stimuli had a duration of $200 \mathrm{~ms}$ (including $10 \mathrm{~ms}$ rise and $40 \mathrm{~ms}$ fall time; sound pressure level $75 \mathrm{~dB}$ ) and were presented with a constant offset-to-onset interval of $600 \mathrm{~ms}$. EEG recordings were identical to Experiment 1.

\subsubsection{Data analysis}

ERPs were computed for each subject and each electrode. All ERP averages were aligned to a $200 \mathrm{~ms}$ baseline before the onset of the target tones. Trials with ocular and amplifier saturation artifacts were excluded from the averages $(13.5 \%)$

The statistical analysis was computed in an ANOVA design for the same electrode sites as in Experiment 1 and with a within-subjects factor probability (rare versus often) and a between-subjects factor lesion (basal ganglia involved versus not involved) on the averages in a time window between 300 and $600 \mathrm{~ms}$.

\section{Results}

Figs. 1c,d display the ERP patterns from the onset of the target tone up to $800 \mathrm{~ms}$ thereafter for each of the two lesion groups at electrode Pz. Fig. 1c shows the ERPs for the patients without basal ganglia lesions, Fig. 1d for the patients with a lesion including the basal ganglia. As can be seen from the figures, both groups show a clear P300 response.

The global ANOVA for the oddball effect in the P300 time window (300-600 ms) at electrode $\mathrm{Pz}$ revealed a main effect of probability $(F(1,12)=19.9, P<0.001)$, but no interaction of probability $\times$ lesion $(F<1)$. Single comparisons revealed that a $\mathrm{P} 300$ effect was found for the patients without $(F(1,6)=6.4, P<0.05)$ as well as for those with a lesion involving the basal ganglia $(F(1,6)=35.9$, $P<0.01)$.

Over the posterior ROIs, there was also main effect of probability $(F(1,12)=30.1, P<0.001)$, but again no interaction of probability $\times$ lesion $(F<1)$. Again, a P300 effect was found for the patients without $(F=10.8$, $P<0.05)$ as well as for those with a lesion including the basal ganglia $(F=32.4, P<0.01)$.

Furthermore, there was an interaction probability $\times$ hemisphere $(F(1,12)=11.8, P<0.01)$ due to the fact that the $\mathrm{P} 300$ effect was stronger in the right-posterior $(F(1,12)=52.9, P<0.001)$ than in the left-posterior ROI $(F(1,12)=13.7, P<0.01)$.

\section{Discussion}

Taken together, the data from the two experiments shed clarifying light onto the question as to which brain structures are involved in the elicitation of the P300 and the P600 as well as onto the relationship between the two components.

Two groups of patients, one with a lesion involving the basal ganglia as well as one group in which this brain area was intact, were tested in an auditory ERP experiment with morphosyntactic violations as well as in an auditory oddball paradigm. As could be demonstrated, a P600 for a grammatical violation was found only in the group with intact basal ganglia, but was not shown by patients with lesions including the basal ganglia. A P300 in response to a rarely occurring auditory stimulus, however, was shown in both groups of patients.

With respect to the question about the neural correlates of the two components these results indicate a single dissociation between the P600 and the P300. More precisely, the basal ganglia seem to play a necessary — though possibly not sufficient - role in the generation or the mediation of the P600, but not of the P300. In other words, we cannot conclude that the basal ganglia necessarily generate the P600 component, since it is also possible that they are part of a network and responsible for transferring information either to or from the actual generator.

With respect to the neuronal sources of the P600, the present results are in agreement with the recent finding that the P600 is strongly modulated in Parkinson patients, that is patients with a degenerative function of the basal ganglia (Friederici et al., 2001). With respect to the ongoing debate about the P600 and P300 in ERP research, the current results have strong implications. The P600 normally found for a broad range of syntactic anomalies appears not to be 'just' a P300-like component in the sense that it reflects the same process of detecting a task-relevant and unexpected event. The finding that the generation of the P600 is dependent on brain structures which do not play a role in eliciting a P300 response is evidence for the assumption that we deal with two different components, seeing that 'at least partially' different generators imply different components as a criterion of component definition (Coles and Rugg, 1995).

\section{Acknowledgements}

We want to thank Anja Hahne, Korinna Eckstein, Juliane Hofmann and Angelika Wolf for their assistance in prepar- 
ing the sentence materials, Claudia Misch for speaking the sentences and Ina Koch for recording the ERP data.

\section{References}

Baayen RH, Piepenbrock R, van Rijn H. The CELEX lexical database, Philadelphia, PA: Linguistic Data Consortium, University of Pennsylvania, 1993.

Coles MGH, Rugg MD. Event-related brain potentials: an introduction. In: Rugg MD, Coles MGH, editors. Electrophysiology of mind: eventrelated potentials and cognition, New York: Oxford University Press, 1995. pp. 1-26.

Coulson S, King J, Kutas M. Expect the unexpected: event-related brain response to morphosyntactic violations. Lang Cogn Proc 1998a;13:2158.

Coulson S, King J, Kutas M. ERPs and domain specificity: beating a straw horse. Lang Cogn Proc 1998b;13:653-672.

Donchin E. Surprise ... surprise? Psychophysiology 1981;18:493-513.

Friederici AD. The time course of syntactic activation during language processing: a model based on neuropsychological and neurophysiological data. Brain Lang 1995;50:259-281.

Friederici AD, Frisch S. Verb-argument structure processing: the role of verb-specific and argument-specific information. J Mem Lang 2000;43:476-507.

Friederici AD, Steinhauer K, Frisch S. Lexical integration: sequential effects of syntactic and semantic information. Mem Cognit 1999;27:438-453.

Friederici AD, Kotz SA, Werheid K, Hein G, von Cramon DY. Selective vulnerability of early and late syntactic processes in Parkinson patients: ERP evidence. Brain Lang 2001;79:15-16.

Frisch S, Schlesewsky M. The N400 reflects problems of thematic hierarchizing. Neuroreport 2001;12:3391-3394.
Gunter TC, Stowe LA, Mulder G. When syntax meets semantics. Psychophysiology 1997;34:660-676.

Hagoort P, Brown C, Groothusen J. The syntactic positive shift as an ERPmeasure of syntactic processing. Lang Cogn Proc 1993;8:439-483.

Hahne A, Friederici AD. Electrophysiological evidence for two steps in syntactic analysis: early automatic and late controlled processes. J Cogn Neurosci 1999;11:194-205.

Johnson Jr R. Auditory and visual P300s in temporal lobectomy patients: evidence for modality-dependent generators. Psychophysiology 1989;26:633-650.

Johnson Jr R. On the neural generators of the P300 component of the eventrelated potential. Psychophysiology 1993;30:90-97.

Mecklinger A, Schriefers H, Steinhauer K, Friederici AD. Processing relative clauses varying on syntactic and semantic dimensions: an analysis with event-related potentials. J Mem Lang 1995;2:477-494.

Mecklinger A, Maess B, Opitz B, Pfeifer E, Cheyne D, Weinberg H. A MEG analysis of the P300 in visual discrimination tasks. Electroenceph clin Neurophysiol 1998;108:45-56.

Menon V, Ford JM, Lim KO, Glover GH, Pfefferbaum A. Combined eventrelated fMRI and EEG evidence for temporal-parietal cortex activation during target detection. Neuroreport 1997;8:3029-3037.

Osterhout L, Hagoort P. A superficial resemblance does not necessarily mean you are part of the family: counterarguments to Coulson, King and Kutas (1998) in the P600/SPS-P300 debate. Lang Cogn Proc 1999;14:1-14.

Osterhout L, Holcomb PJ. Event-related potentials and syntactic anomaly. J Mem Lang 1992;31:785-804.

Picton TW. The P300 wave of the human event-related potential. J Clin Neurophysiol 1992;9:456-479.

Rogers RL, Baumann SB, Papanicolaou AC, Bourbon TW, Alagarsamy S, Eisenberg HM. Localization of the P3 sources in using magnetoencephalography and magnetic resonance imaging. Electroenceph clin Neurophysiol 1991;79:308-321. 